\title{
Multiple Solutions for Generalized Asymptotical Linear Hamiltonian Systems Satisfying Bolza Boundary Conditions
}

\author{
Yuan Shan ${ }^{1,2}$ and Baoqing Liu' \\ ${ }^{1}$ School of Mathematical Sciences, Nanjing Normal University, Nanjing, Jiangsu 210023, China \\ ${ }^{2}$ Department of Mathematics, Nanjing University, Nanjing, Jiangsu 210093, China
}

Correspondence should be addressed to Yuan Shan; shannjnu@yahoo.cn

Received 20 December 2012; Accepted 28 February 2013

Academic Editor: Jinde Cao

Copyright (c) 2013 Y. Shan and B. Liu. This is an open access article distributed under the Creative Commons Attribution License, which permits unrestricted use, distribution, and reproduction in any medium, provided the original work is properly cited.

\begin{abstract}
This paper is devoted to multiple solutions of generalized asymptotical linear Hamiltonian systems satisfying Bolza boundary conditions. We classify the linear Hamiltonian systems by the index theory and obtain the existence and multiplicity of solutions for the Hamiltonian systems, based on an application of the classical symmetric mountain pass lemma.
\end{abstract}

\section{Introduction}

This paper is concerned with a classical problem on the existence of solutions for Hamiltonian systems when a more general form of twist condition between the origin and infinity holds for the Hamiltonian function. More precisely consider the system

$$
\begin{gathered}
\dot{x}=J H^{\prime}(t, x), \\
P x(0)=0=P x(1),
\end{gathered}
$$

where $H \in C\left(\mathbf{R} \times \mathbf{R}^{2 n}, \mathbf{R}\right)$, and $H^{\prime} \in C\left(\mathbf{R} \times \mathbf{R}^{2 n}, \mathbf{R}^{2 n}\right)$ and $H^{\prime}$ satisfies the following:

$\left(H_{1}\right) H^{\prime}(t, x)=B_{0}(t, x) x+o(|x|)$, as $|x| \rightarrow 0$ uniformly in $t \in[0,1]$,

$\left(H_{2}\right) H^{\prime}(t, x)=B_{\infty}(t, x) x+o(|x|)$, as $|x| \rightarrow \infty$ uniformly in $t \in[0,1]$,

for $B_{0}(t, x), B_{\infty} \in L^{\infty}\left((0,1) \times \mathbf{R}^{2 n} ; \operatorname{GLs}\left(\mathbf{R}^{2 n}\right)\right)$. Here and below, we use $H^{\prime}$ to denote the first derivative of $H$ with respect to $x \in \mathbf{R}^{2 n}$.

A quantitative way to measure the twisting is given by the Maslov-type index. As in [1,2], an index for the second-order and first-order linear Hamiltonian systems was defined and developed in [3] for the study of linear operator equation with infinite Morse index. In $[4,5]$, by Conley and Zehnder and
Long, an index theory for symplectic path was defined. We refer to two excellent books $[6,7]$ for systematical treatment.

In [2], Dong discussed the classification of the linear Hamiltonian systems with Bolza boundary conditions as follows:

$$
\begin{gathered}
\dot{x}=J B(t) x, \\
P x(0)=0=P x(1),
\end{gathered}
$$

where $B \in L^{\infty}\left((0,1) ; \operatorname{GLs}\left(\mathbf{R}^{2 n}\right)\right)$ and $P x=\left(x_{1}, \ldots x_{n}\right) \in$ $\mathbf{R}^{n}$, for any $x=\left(x_{1}, \ldots, x_{2 n}\right)$. That is, for any $B \in$ $L^{\infty}\left((0,1) ; \operatorname{GLs}\left(\mathbf{R}^{2 n}\right)\right)$, he associated it with a pair of numbers $(i(B), v(B)) \in Z \times\{0,1, \ldots, n\}$. This pair of integers is called index and nullity of $B$, respectively. And he defined the nullity $\nu(B)$ as the dimension of the solution space of (1). Let $E[\alpha]$ be the integer $a \in \mathbf{Z}$ such that $a<\alpha \leq a+1$. So that $E[a]=a-1$ for all integer $a$ defined as in [6]. In order to process the definition of $i(B)$, he defined $i\left(\lambda I_{2 n}\right)=n E[\lambda / \pi]$. In particular, when $\lambda \in(k \pi,(k+1) \pi), i\left(\lambda I_{2 n}\right)=n k$. We will introduce the definitions and properties of $i(B)$ in detail in Section 2. After the discussion of the index theory, we will prove our main result in Section 3.

Throughout this paper, $\|x\|_{C}$ denotes the usual norm in $C[0,1]$. For any $A_{1}, A_{2} \in \operatorname{GLs}\left(\mathbf{R}^{2 n}\right)$, we write $A_{1} \leq A_{2}$ if $A_{2}-$ $A_{1}$ is positively semidefinite, and we write $A_{1}<A_{2}$ if $A_{2}-A_{1}$ is positive definite. For any $A_{1}, A_{2} \in L^{\infty}\left((0,1) ; \operatorname{GLs}\left(\mathbf{R}^{2 n}\right)\right)$, we write $A_{1} \leq A_{2}$ if $A_{1}(t) \leq A_{2}(t)$ for a.e. $t \in(0,1)$, and we write 
$A_{1}<A_{2}$ if $A_{1} \leq A_{2}$ and $A_{1}(t)<A_{2}(t)$ on a subset of $(0,1)$ with a nonzero measure.

Remark 1. Let $C_{1}, C_{2} \in(k \pi,(k+1) \pi), C_{3}, C_{4} \in((k+1) \pi,(k+$ 2) $\pi$ ) with $C_{1}<C_{2}, C_{3}<C_{4}$. Assume that $A_{i}=C_{i} I_{2 n}, i=1,2$, and $B_{i}=C_{i} I_{2 n}, i=3,4$. Then, we have $i\left(A_{1}\right)=i\left(A_{2}\right)=n k$, $i\left(B_{1}\right)=i\left(B_{2}\right)=n(k+1)$, and $\nu\left(A_{i}\right)=\nu\left(B_{i}\right)=0$, for $i=1,2$; (1) has at least $n$ pairs of solutions.

We make use of the critical point theory $[8,9]$ to prove Theorem 8. The novelty of our result is that it suffices to assume that $H(t, x)+(1 / 2) \mu|x|^{2}$ is convex. The twisting between origin and infinity is reflected in $\left(H_{1}^{*}\right)$ and $\left(H_{2}^{*}\right)$. Thus, our results complement with Theorem 3.8 in [2] and Theorem 1.1 in [10]. For other results, we refer to [11-15].

This paper is organized as follows. In Section 2, we introduce some preliminaries including index theory, and establish the $\mu$-index theory which is needed in the proofs. In Section 3, we present the proofs of the results.

\section{Index Theory for Linear Hamiltonian Systems Satisfying Bolza Boundary Value Conditions}

First, we recall some definitions and propositions in [2]. We consider the following system:

$$
\begin{gathered}
\dot{x}=J B(t) x, \\
P x(0)=0=P x(1) .
\end{gathered}
$$

Let $H=\left\{x:[0,1] \rightarrow \mathbf{R}^{2 n} \mid x\right.$ is continuous on $[0,1]$, satisfies (4), and $\left.\dot{x} \in L^{2}\right\}$ with the norm $\|x\|_{H}=\left(\int_{0}^{1}\left(\left|x^{\prime}(t)\right|^{2}+\right.\right.$ $\left.\left.|x(t)|^{2}\right) d t\right)^{1 / 2},\left(\Lambda_{1} x\right)(t)=J x^{\prime}(t),(\bar{B} x)(t)=B(t) x(t)$, where $L^{2}:=L^{2}\left((0,1) ; \mathbf{R}^{2 n}\right)$, and let $|x|=\left(\sum_{i=1}^{2 n}\left|x_{i}\right|\right)^{1 / 2}$ for any $x=$ $\left(x_{1}, \ldots, x_{2 n}\right) \in \mathbf{R}^{2 n}$. Then, $\Lambda_{1}$ and $\bar{B}$ are self-adjoint operators, and $\bar{B}$ is bounded.

Definition 2 (see [2, Definitions 2.1, 2.3, 2.4, and 2.7]). For any $B_{1}, B_{2}, B \in L^{\infty}\left((0,1), G L s\left(\mathbf{R}^{2 n}\right)\right)$, one defines that

(1) $v(B)=\operatorname{dim} \operatorname{ker}\left(\Lambda_{1}+\bar{B}\right)$,

(2) $I\left(B_{1}, B_{2}\right)=\sum_{\lambda \in[0,1)} \nu\left((1-\lambda) B_{1}+\lambda B_{2}\right)$ for $B_{1}<B_{2}$,

(3) $i(B)=i\left(\lambda I_{2 n}\right)-I\left(B, \lambda I_{2 n}\right)$ for $\lambda \in \mathbf{R}$ satisfies $B<\lambda I_{2 n}$.

Using spectral theory, a Morse-type index $i_{\mu}(B)$ was established in [2]. More precisely, for any $B \in L^{\infty}((0,1)$, $\left.\operatorname{GLs}\left(\mathbf{R}^{2 n}\right)\right)$, let $\mu \in \mathbf{R} \backslash \pi \mathbf{Z}$ with $B+\mu I_{2 n} \geq I_{2 n}$. Then, $\nu\left(-\mu I_{2 n}=0\right), \Lambda x:=J \dot{x}(t)-\mu x(t)$ is invertible, and the inverse $\Lambda^{-1}: L^{2} \rightarrow L^{2}$ is self-adjoint and compact. He put a quadratic form:

$$
\begin{array}{r}
q_{\mu, B}(u, u)=\frac{1}{2} \int_{0}^{1}\left(\Lambda^{-1} u(t), u(t)\right)+\left(C_{\mu}(t) u(t), u(t)\right) d t \\
\forall u \in L^{2} \\
\left(C_{\mu}(t) u(t), u(t)\right):=\int_{0}^{1}\left(C_{\mu}(t) u(t), u(t)\right),
\end{array}
$$

where $C_{\mu}(t):=\left(\mu I_{2 n}+B(t)\right)^{-1}$. Then, $\left(\bar{C}_{\mu}(t) u(t), u(t)\right)$ defines a Hilbert space structure on $L^{2} . C_{\mu}^{-1} \Lambda^{-1}$ is a self-adjoint and compact operator under this interior product. By the spectral theory, there is a basis $e_{j}, j \in \mathbf{N}$ of $L^{2}$, and a sequence $\lambda_{j} \rightarrow 0$ in $\mathbf{R}$ such that

$$
\begin{gathered}
\left(C_{\mu}(t) e_{i}(t), e_{j}(t)\right)=\delta_{i j}, \\
\left(\Lambda^{-1} e_{j}, u\right)=\left(C_{\mu} \lambda_{j} e_{j}, u\right) \quad \forall u \in L^{2} .
\end{gathered}
$$

For any $u \in L^{2}$ as $u=\sum_{j=1}^{\infty} \xi_{j} e_{j}$, (5) can be rewritten as follows:

$$
\begin{aligned}
q_{\mu, B}(u, u) & =\frac{1}{2} \int_{0}^{1}\left(\Lambda^{-1} u, u\right)+\left(C_{\mu}(t) u, u\right) d t \\
& =\frac{1}{2} \sum_{j=1}^{\infty}\left(1+\lambda_{j}\right) \xi_{j}^{2} .
\end{aligned}
$$

Define that

$$
\begin{aligned}
& E_{\mu}^{-}(B):=\left\{\sum_{j=1}^{\infty} \xi_{j} e_{j} \mid \xi_{j}=0 \text { if } 1+\lambda_{j} \geq 0\right\}, \\
& E_{\mu}^{0}(B):=\left\{\sum_{j=1}^{\infty} \xi_{j} e_{j} \mid \xi_{j}=0 \text { if } 1+\lambda_{j} \neq 0\right\}, \\
& E_{\mu}^{+}(B):=\left\{\sum_{j=1}^{\infty} \xi_{j} e_{j} \mid \xi_{j}=0 \text { if } 1+\lambda_{j} \leq 0\right\} .
\end{aligned}
$$

$E_{\mu}^{-}(B), E_{\mu}^{0}(B)$, and $E_{\mu}^{+}(B)$ are $q_{\mu, B}$ orthogonal, and $E_{\mu}^{-}(B) \oplus$ $E_{\mu}^{0}(B) \oplus E_{\mu}^{+}(B)=L^{2}$. Since $\lambda_{j} \rightarrow 0$ as $j \rightarrow \infty, E_{\mu}^{-}(B)$ and $E_{\mu}^{0}(B)$ are two finite-dimensional subspaces.

Definition 3 (see [2, Definition 2.9]). For any $B \in L^{\infty}((0,1)$, $\left.\operatorname{GLs}\left(\mathbf{R}^{2 n}\right)\right), \mu \in \mathbf{R}$ with $\mu I_{2 n}+B \geq I_{2 n}$, one defines that

$$
\nu_{\mu}(B):=\operatorname{dim} E_{\mu}^{0}(B), \quad i_{\mu}(B):=\operatorname{dim} E_{\mu}^{-}(B) .
$$

One calls $\nu_{\mu}(B)$ and $i_{\mu}(B) \mu$-nullity and $\mu$-index of $B$, respectively. 
Proposition 4 (see [2, Propositions 2.10, 2.13]). The index, relative Morse index, and $\mu$-index have the following properties.

(1) For any $B_{1}, B_{2}, B \in L^{\infty}\left((0,1), G L s\left(\mathbf{R}^{2 n}\right)\right)$, with $B_{1}<$ $B_{2}$, one has

$$
\begin{gathered}
\nu_{\mu}(B)=\nu(B), \quad I\left(B_{1}, B_{2}\right)=i_{\mu}\left(B_{2}\right)-i_{\mu}\left(B_{1}\right), \\
i_{\mu}\left(B_{1}\right)-i_{\mu}\left(B_{2}\right)=i\left(B_{1}\right)-i\left(B_{2}\right) .
\end{gathered}
$$

(2) There exists $\epsilon_{0}>0$, such that, for any $\epsilon \in\left(0, \epsilon_{0}\right]$, one gets

$$
\begin{gathered}
\nu\left(B+\epsilon I_{2 n}\right)=0=v\left(B-\epsilon I_{2 n}\right), \\
i\left(B-\epsilon I_{2 n}\right)=i(B), \quad i\left(B+\epsilon I_{2 n}\right)=i(B)+v(B) .
\end{gathered}
$$

In particular, if $\nu(B)=0$, one obtains $i\left(B+\epsilon I_{2 n}\right)=i(B)$ for $\epsilon \in\left(0, \epsilon_{0}\right]$.

(3) $i_{\mu}(B)-i(B)$ is a constant for $B$ satisfying $B+\mu I_{2 n} \geq$ $I_{2 n}$, that is, $i_{\mu}(B)-i(B)=i_{\mu}\left(B_{1}\right)-i\left(B_{1}\right)$ for any other $B_{1} \in L^{\infty}\left((0,1), G L s\left(\mathbf{R}^{2 n}\right)\right)$ with $B_{1}+\mu I_{2 n} \geq I_{2 n}$. For any $\mu>1$, one has

$$
\begin{gathered}
i_{\mu}(0)=n E\left[\frac{\mu}{\pi}\right], \\
i_{\mu}(B)=n E\left[\frac{\mu}{\pi}\right]+n+i(B),
\end{gathered}
$$

for any $B \in L^{\infty}\left((0,1), G L s\left(\mathbf{R}^{2 n}\right)\right)$ with $B+\mu I_{2 n} \geq I_{2 n}$.

Remark 5. From this proposition, there is $\epsilon_{0}>0, \epsilon \in\left(0, \epsilon_{0}\right]$ such that $\nu_{\mu}\left(B+\epsilon I_{2 n}\right)=0=\nu_{\mu}\left(B-\epsilon I_{2 n}\right)$ and $i_{\mu}\left(B-\epsilon I_{2 n}\right)=$ $i_{\mu}(B), i_{\mu}\left(B+\epsilon I_{2 n}\right)=i_{\mu}(B)+\nu_{\mu}(B)$.

In order to prove Theorem 8 , we make use of minimax arguments for the multiplicity of critical points in the presence of symmetry. We state two results of this type from $[8,9]$.

Lemma 6 (cf. Chang [9, Theorem 4.3.4]). Assume that $f \in$ $C^{1}\left(X, \mathbf{R}^{1}\right)$ satisfies the $(P S)$ condition, $f(\theta)=0, f(-x)=f(x)$, and

(1) there are an $m$-dimensional subspace $X_{1}$ and a constant $\rho>0$ such that

$$
\sup _{x \in X_{1} \cap S_{\rho}} f(x)<0
$$

(2) there is a j-dimensional subspace $X_{2}$ such that

$$
\inf _{x \in X_{2}^{\perp}} f(x)>-\infty
$$

where $X_{2}^{\perp}$ is a subset of $X$ such that $X_{2}^{\perp} \oplus X_{2}=X$. Then, $f$ has at least $m-j$ pairs of critical points if $m-j>0$.

Lemma 7 (see [8, Theorem 2.2.8]). Suppose that $f \in$ $C^{\prime}(H, \mathbf{R})$ satisfies $(C)$ condition, $f(\theta) \geq 0$, even, and there exist two closed subspaces $\mathrm{H}^{+} \mathrm{H}^{-}$of $\mathrm{H}$, with codim $\mathrm{H}^{-}<+\infty$, and two constants $c_{\infty}>c_{0} \geq f(0)$ such that

(a) $f(u) \geq c_{0}$ for all $u \in S_{\rho} \cap H^{+}$,

(b) $f(u) \leq c_{\infty}$ for all $u \in H^{-}$.

Then, if $\operatorname{dim} H^{-} \geq \operatorname{codim} H^{+}, f$ possesses at least $m=$ $\operatorname{dim} \mathrm{H}^{-}-\operatorname{codim} \mathrm{H}^{+}$distinct pairs of critical points whose corresponding critical values belong to $\left[c_{0}, c_{\infty}\right]$.

\section{Proof of Main Results}

We state the main result in this paper. We further make the following assumptions.

$\left(H_{1}^{*}\right)$ There exists $A_{1}, A_{2} \in L^{\infty}\left((0,1) ; \operatorname{GLs}\left(\mathbf{R}^{2 n}\right)\right)$ such that $A_{1}(t) \leq B_{0}(t, x) \leq A_{2}(t)$ with $i\left(A_{1}\right)=i\left(A_{2}\right), v\left(A_{2}\right)=$ 0 .

$\left(H_{2}^{*}\right)$ There exists $B_{1}, B_{2} \in L^{\infty}\left((0,1) ; \operatorname{GLs}\left(\mathbf{R}^{2 n}\right)\right)$ such that $B_{1}(t) \leq B_{\infty}(t, x) \leq B_{2}(t)$ with $i\left(B_{1}\right)=i\left(B_{2}\right), v\left(B_{2}\right)=$ 0 .

$\left(H_{3}\right) H(t, \theta)=0, H(t, x)=H(t,-x)$.

$\left(H_{4}\right) H(t, x)+(1 / 2) \mu|x|^{2}$ is convex.

Our main result reads as follows.

Theorem 8. Assume that $\left(H_{1}\right),\left(H_{1}^{*}\right),\left(H_{2}\right),\left(H_{2}^{*}\right),\left(H_{3}\right),\left(H_{4}\right)$ are satisfied, then (1) has at least $\left|i\left(A_{1}\right)-i\left(B_{1}\right)\right|$ pairs of solutions.

Theorem 9. If $i\left(A_{1}\right)>i\left(B_{1}\right)$, then (1) has at least $i\left(A_{1}\right)-i\left(B_{1}\right)$ pairs of solutions.

If $i\left(A_{1}\right)>i\left(B_{1}\right)$, let $\mu \in \mathbf{R} \backslash \pi \mathbf{Z}$ with $A_{1}+\mu I_{2 n} \geq I_{2 n}$, $B_{1}+\mu I_{2 n} \geq I_{2 n}$. Recalling that $\Lambda x=J \dot{x}-\mu I_{2 n}$, one denotes $H_{\mu}(t, x)=H(t, x)+(1 / 2) \mu|x|^{2}$. Then, one obtains

$$
H_{\mu}^{*}(t, x)=\sup _{y \in \mathbf{R}^{2 n}}\left\{(x, y)-H_{\mu}(t, y)\right\}
$$

Thus, $H_{\mu}^{*}(t,-x)=H^{*}(t, x)$ and

$$
x=H_{\mu}^{* \prime}\left(t, x^{*}\right) \quad \text { iff } x^{*}=H_{\mu}^{\prime}(t, x)
$$

(by the Fenchel conjugate formula; see [6, Proposition II]). Hence, $|x| \rightarrow \infty$ if and only if $\left|x^{*}\right| \rightarrow \infty$. Consider the functional defined by

$$
\psi(u)=\int_{0}^{1}\left(\frac{1}{2} \Lambda^{-1} u(t), u(t)\right)+H_{\mu}^{*}(t, u(t)) d t
$$

$\forall u \in L^{2}$

In order to prove Theorem 9, we need the following lemmas.

Lemma 10. $\psi$ satisfies the (PS) condition. 
Proof. Assume that $\left\{u_{j}\right\}$ is a sequence in $L^{2}$ such that $\psi\left(u_{j}\right)$ is bounded and $\psi^{\prime}\left(u_{j}\right) \rightarrow 0$ in $L^{2}$. It suffices to prove that $\left\{u_{j}\right\}$ has convergent sequence. By (18), we have

$$
\left(\psi^{\prime}(u), v\right)=\int_{0}^{1}\left(\Lambda^{-1} u(t), v(t)\right)+\left(H_{\mu}^{* \prime}(t, u(t)), v(t)\right) d t,
$$

for all $v \in L^{2}$. Hence, we get

$$
\psi^{\prime}\left(u_{j}\right)=\Lambda^{-1} u_{j}+H_{\mu}^{* \prime}\left(t, u_{j}(t)\right) \longrightarrow \theta, \quad \text { in } L^{2} .
$$

Let $x_{j}=\Lambda^{-1} u_{j}, y_{j}=u_{j} /\left\|x_{j}\right\|_{C}$, and $z_{j}=\psi^{\prime}\left(u_{j}\right)-\Lambda^{-1}\left(u_{j}\right)$. If $\left\|x_{j}\right\|_{C} \rightarrow \infty$, then $\left\|x_{j}\right\|_{L^{2}} \rightarrow \infty$. Joining $\left(H_{2}\right)$ with (17) and (20), we have

$$
\begin{aligned}
u_{j}(t)= & H_{\mu}^{\prime}\left(t, z_{j}(t)\right) \\
= & \left(B_{\infty}\left(t, z_{j}(t)\right)+\mu I_{2 n}\right) z_{j}(t) \\
& +o\left(\left|z_{j}(t)\right|\right) \\
= & -\left(B_{\infty}\left(t, z_{j}(t)\right)+\mu I_{2 n}\right) \Lambda^{-1} u_{j} \\
& +\left(B_{\infty}\left(t, z_{j}(t)\right)+\mu I_{2 n}\right) \psi^{\prime}\left(u_{j}\right) \\
& +o\left(\left|z_{j}(t)\right|\right) .
\end{aligned}
$$

Using the preceding notations, we have

$$
\begin{aligned}
y_{j}(t)= & -\left(B_{\infty}\left(t, z_{j}(t)\right)+\mu I_{2 n}\right) \Lambda^{-1} y_{j} \\
& +\left(B_{\infty}\left(t, z_{j}(t)\right)+\mu I_{2 n}\right) \psi^{\prime}\left(u_{j}\right)\left\|x_{j}\right\|_{C}^{-1} \\
& +o\left(\left|z_{j}(t)\right|\right)\left\|x_{j}\right\|_{C}^{-1} .
\end{aligned}
$$

So $y_{j}(t)$ is bounded in $L^{2}$. We assume that $y_{j}-\bar{y}$ in $L^{2}$, and hence $\Lambda^{-1} y_{j} \rightarrow \Lambda^{-1} \bar{y}$. From (22), there exists $\bar{B} \in$ $L^{\infty}\left((0,1) ; \operatorname{GLs}\left(\mathbf{R}^{2 n}\right)\right)$, such that

$$
B_{\infty}\left(t, z_{j}(t)\right) v(t) \rightarrow \bar{B}(t) v(t) \quad \text { for any } v \in L_{2} .
$$

And we have $B_{1}(t) \leq \bar{B}(t) \leq B_{2}(t)$. Taking the limit as $j \rightarrow \infty$ in (22), we obtain $\bar{y}(t)=-\left(\bar{B}(t)+\mu I_{2 n}\right) \Lambda^{-1} \bar{y}$. Let $\bar{x}=\Lambda^{-1} \bar{y}$. We get

$$
J \bar{x}+\bar{B}(t) \bar{x}=0, \quad P \bar{x}(0)=0=P \bar{x}(1) .
$$

By assumption $\left(H_{2}^{*}\right)$, we have $i(\bar{B})=0$ and $\bar{x}=0$, which is impossible. Since $\|\bar{x}\|_{C}=1,\left\|\Lambda^{-1} u_{j}\right\|_{C}$ is bounded. From (17) and (20), $\left\|u_{j}\right\|_{L^{2}}$ is bounded. Assume that $u_{j} \rightarrow u_{0}$ in $L^{2}$; then $\Lambda^{-1} u_{j} \rightarrow \Lambda^{-1} u_{0}$. Let $\xi_{j}:=\Lambda^{-1} u_{j}+H_{\mu}^{* \prime}\left(t, u_{j}\right)$; then $H_{\mu}^{* \prime}\left(t, u_{j}\right)=\xi_{j}-\Lambda^{-1} u_{j} \rightarrow-\Lambda^{-1} u_{0}$. Fenchel conjugate formula gives $u_{j}=H_{\mu}^{* \prime}\left(t, \xi_{j}-\Lambda^{-1} u_{j}\right) \rightarrow H_{\mu}^{* \prime}\left(t,-\Lambda^{-1} u_{0}\right)$ in $L^{2}$ (by [6, Preposition II, Theorem 4]).

Lemma 11. The assumption (1) of Lemma 6 is valid, where $f$ is defined as in (18).
Proof. From $\left(H_{1}\right)$ and $\left(H_{1}^{*}\right)$, we have that, for any $\epsilon>0$, there exists $\delta>0$ such that

$$
\left(\left(A_{1}(t)-\epsilon\right) x, x\right) \leq\left(H^{\prime}(t, x), x\right) \leq\left(\left(A_{2}(t)+\epsilon\right) x, x\right),
$$

for any $|x| \leq \delta$.

Thus,

$$
\begin{aligned}
H_{\mu}(t, y) & =\left(\int_{0}^{1} H_{\mu}^{\prime}(t, \theta y) d \theta, y\right) \\
& \geq \frac{1}{2}\left(\left(\left(A_{1}(t)-\epsilon I_{2 n}\right)+\mu I_{2 n}\right) y, y\right),
\end{aligned}
$$

for any $|y| \leq \delta$.

Let $g(y)=(x, y)-H_{\mu}(t, y)$. By $(16)$ and $\left(H_{4}\right), g(y)$ is strictly concave. Then, $|y| \rightarrow \infty$ as $g(y) \rightarrow-\infty$. So, $g$ achieves its maximum at a unique point. Because of $g^{\prime}(y)=$ $x-H_{\mu}^{\prime}(t, y)$, we get that

$$
x=H_{\mu}^{\prime}(t, y) \text { has a unique solution. }
$$

And we can easily get $0=H_{\mu}^{\prime}(t, 0)$. Hence, we have $|y| \rightarrow$ 0 when $|x| \rightarrow 0$. Otherwise, there exists $\epsilon_{0}>0, x_{n}, y_{n} \in \mathbf{R}^{2 n}$, $t_{n} \in[0,1]$ s.t. $\left|x_{n}\right| \rightarrow 0$. But $\left|y_{n}\right| \geq \epsilon_{0}, x_{n}=H_{\mu}^{\prime}\left(t_{n}, y_{n}\right)$. Hence, $\left|y_{n}\right|$ is bounded, and there exists $y_{0}, t_{0}$ such that $y_{n} \rightarrow y_{0}$, $t_{n} \rightarrow t_{0}, x_{n} \rightarrow 0$. Thus, $0=H_{\mu}^{\prime}\left(t_{0}, y_{0}\right)$. This contradicts the uniqueness, which yields there exists $\rho(\rho<\delta)$ such that, for any $|x| \leq \rho$, we have $|y| \leq \delta$. So, for any $|x| \leq \rho$, we have

$$
\begin{aligned}
H_{\mu}^{*}(t, x) & =\sup _{y \in \mathbf{R}^{2 n}}\left\{(x, y)-H_{\mu}(t, y)\right\} \\
& =\sup _{|y| \leq \delta}\left\{(x, y)-H_{\mu}(t, y)\right\} \\
& \leq \sup _{|y| \leq \delta}\left\{(x, y)-\frac{1}{2}\left(\left(\left(A_{1}(t)-\epsilon I_{2 n}\right)+\mu I_{2 n}\right) y, y\right)\right\} \\
& =\frac{1}{2}\left(\left(\left(A_{1}(t)-\epsilon I_{2 n}\right)+\mu I_{2 n}\right)^{-1} x, x\right) .
\end{aligned}
$$

By (18),

$$
\begin{aligned}
\psi(u) \leq \int_{0}^{1} & \left(\frac{1}{2} \Lambda^{-1} u(t), u(t)\right) \\
& +\frac{1}{2}\left(\left(\left(A_{1}(t)-\epsilon I_{2 n}\right)+\mu I_{2 n}\right)^{-1} u(t), u(t)\right) d t
\end{aligned}
$$$$
\text { for any }|u| \leq \rho \text {, }
$$

that is,

$$
\psi(u) \leq q_{\mu,\left(A_{1}-\epsilon\right)}(u, u) \quad \text { for any }|u| \leq \rho .
$$

Let $X_{1}=E_{\mu}^{-}\left(A_{1}-\epsilon\right)$. Then, $\operatorname{dim} X_{1}=i_{\mu}\left(A_{1}-\epsilon\right)$. For any $u \in X_{1} \cap S_{\rho}$, we have sup $\psi(u)<0$. By Proposition 4 , for $\epsilon$ being small enough, $i_{\mu}\left(A_{1}-\epsilon\right)=i_{\mu}\left(A_{1}\right)$. 
Lemma 12. The assumption (2) of Lemma 6 is also satisfied.

Proof. From $\left(H_{2}\right)$, let $h_{2}(t, x)=H^{\prime}(t, x)-B_{\infty}(t, x) x$. Then, for any $\epsilon>0$, there exists a constant $M_{1}$ such that

$$
\left|h_{2}(t, x)\right| \leq \epsilon|x|+M_{1}, \quad \forall(t, x) \in[0,1] \times \mathbf{R}^{2 n} .
$$

Combining $\left(H_{2}\right)$ and (31), there exists a constant $M_{2}$ such that

$$
H_{\mu}(t, x) \leq \frac{1}{2}\left(\left(B_{2}(t)+4 \epsilon I_{2 n}+\mu I_{2 n}\right) x, x\right)+M_{2},
$$

for all $(t, x) \in[0,1] \times \mathbf{R}^{2 n}$. By the definition of $H_{\mu}^{*}(t, x)$, we have

$$
\begin{aligned}
& H_{\mu}^{*}(t, x) \\
& \quad \geq \sup _{y \in \mathbf{R}^{2 n}}\left\{(x, y)-\frac{1}{2}\left(\left(B_{2}(t)+4 \epsilon I_{2 n}+\mu I_{2 n}\right) y, y\right)\right\}-M_{2} \\
& \quad=\frac{1}{2}\left(\left(\left(B_{2}(t)+4 \epsilon I_{2 n}\right)+\mu I_{2 n}\right)^{-1} x, x\right)-M_{2} .
\end{aligned}
$$

Thus,

$$
\begin{aligned}
\psi(u) \geq \int_{0}^{1}( & \left.\frac{1}{2} \Lambda^{-1} u(t), u(t)\right) \\
& +\frac{1}{2}\left(\left(B_{2}(t)+4 \epsilon I_{2 n}\right)+\mu I_{2 n}\right)^{-1}(u(t), u(t)) d t \\
& -M_{2} .
\end{aligned}
$$

So, we infer

$$
\psi(u) \geq q_{\mu,\left(B_{2}+4 \epsilon\right)}(u, u)-M_{2} .
$$

Let $X_{2}=E_{\mu}^{-}\left(B_{2}+4 \epsilon\right)$. We have $\operatorname{dim} X_{2}=i_{\mu}\left(B_{2}+4 \epsilon\right)$ and $X_{2}^{\perp}=E_{\mu}^{0}\left(B_{2}(t)+4 \epsilon\right) \oplus E_{\mu}^{+}\left(B_{2}(t)+4 \epsilon\right)$. So, we have

$$
\inf _{X_{2}^{\perp}} \psi(u) \geq-M_{2} \text {. }
$$

By Proposition 4 , we have $i_{\mu}\left(A_{1}\right)-i_{\mu}\left(B_{2}\right)=i\left(A_{1}\right)-i\left(B_{2}\right)$, and we can also let $\epsilon$ be small enough such that $i_{\mu}\left(B_{2}+4 \epsilon\right)=$ $i_{\mu}\left(B_{2}\right)$. This completes the proof.

Theorem 13. If $i\left(A_{1}\right)<i\left(B_{1}\right)$, then (2) has at least $i\left(B_{1}\right)-$ $i\left(A_{1}\right)$ pairs of solutions.

The argument in Theorem 8 can also be used here by using Lemma 7. More precisely, if $H^{+}=E_{\mu}^{+}\left(A_{2}+\epsilon\right)$, then $\operatorname{codim} H^{+}=i\left(A_{1}\right)$ and $\psi(u)>c_{0}>0$ on $H^{+} \cap S_{\rho}$. If $H^{-}=E_{\mu}^{-}\left(B_{1}-4 \epsilon\right)$, then $\operatorname{dim} H^{-}=i\left(B_{1}\right)$ and $\psi(u) \leq c_{\infty}$ on $H^{-}$.

\section{Acknowledgments}

The author would like to thank the reviewers for their valuable comments which improved the paper. The paper is partially supported by the National Natural Science Foundation of China (10871095) and the Foundation for Innovative Program of Jiangsu Province (CXZZ12_0377, CXZZ12_0386).

\section{References}

[1] Y. Dong, "Index theory, nontrivial solutions, and asymptotically linear second-order Hamiltonian systems," Journal of Differential Equations, vol. 214, no. 2, pp. 233-255, 2005.

[2] Y. Dong, "Maslov type index theory for linear Hamiltonian systems with Bolza boundary value conditions and multiple solutions for nonlinear Hamiltonian systems," Pacific Journal of Mathematics, vol. 221, no. 2, pp. 253-280, 2005.

[3] Y. Dong, "Index theory for linear selfadjoint operator equations and nontrivial solutions for asymptotically linear operator equations," Calculus of Variations and Partial Differential Equations, vol. 38, no. 1-2, pp. 75-109, 2010.

[4] C. Conley and E. Zehnder, "Morse-type index theory for flows and periodic solutions for Hamiltonian equations," Communications on Pure and Applied Mathematics, vol. 37, no. 2, pp. 207253, 1984.

[5] Y. M. Long, "Maslov-type index, degenerate critical points, and asymptotically linear Hamiltonian systems," Science in China Series A, vol. 33, no. 12, pp. 1409-1419, 1990.

[6] I. Ekeland, Convexity Methods in Hamiltonian Mechanics, vol. 19, Springer, Berlin, Germany, 1990.

[7] J. Mawhin and M. Willem, Critical Point Theory and Hamiltonian Systems, vol. 74 of Applied Mathematical Sciences, Springer, New York, NY, USA, 1989.

[8] P. Bartolo, V. Benci, and D. Fortunato, "Abstract critical point theorems and applications to some nonlinear problems with "strong" resonance at infinity," Nonlinear Analysis: Theory, Methods \& Applications, vol. 7, no. 9, pp. 981-1012, 1983.

[9] K. C. Chang, Critical Point Theory and Its Application, Shanghai Kexue Jishu Chubanshe, Shanghai, China, 1986.

[10] Y. Shan, "Multiple solutions of generalized asymptotical linear Hamiltonian systems satisfying Sturm-Liouville boundary conditions," Nonlinear Analysis: Theory, Methods \& Applications, vol. 74, no. 14, pp. 4809-4819, 2011.

[11] V. Benci, "On critical point theory for indefinite functionals in the presence of symmetries," Transactions of the American Mathematical Society, vol. 274, no. 2, pp. 533-572, 1982.

[12] F. Dalbono and C. Rebelo, "Multiplicity of solutions of Dirichlet problems associated with second-order equations in $R^{2}$," Proceedings of the Edinburgh Mathematical Society. Series II, vol. 52, no. 3, pp. 569-581, 2009.

[13] F. Dalbono, "Branches of index-preserving solutions to systems of second order ODEs," Nonlinear Differential Equations and Applications, vol. 16, no. 5, pp. 569-595, 2009.

[14] G. H. Fei, "Relative Morse index and its application to Hamiltonian systems in the presence of symmetries," Journal of Differential Equations, vol. 122, no. 2, pp. 302-315, 1995.

[15] D. Zhang, "Relative Morse index and multiple brake orbits of asymptotically linear Hamiltonian systems in the presence of symmetries," Journal of Differential Equations, vol. 245, no. 4, pp. 925-938, 2008. 


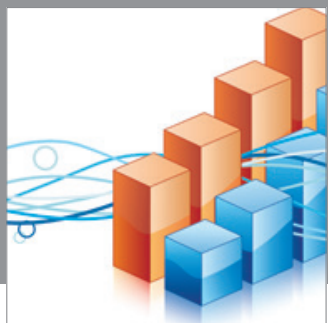

Advances in

Operations Research

mansans

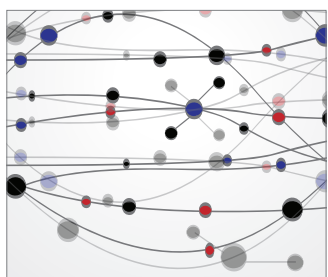

The Scientific World Journal
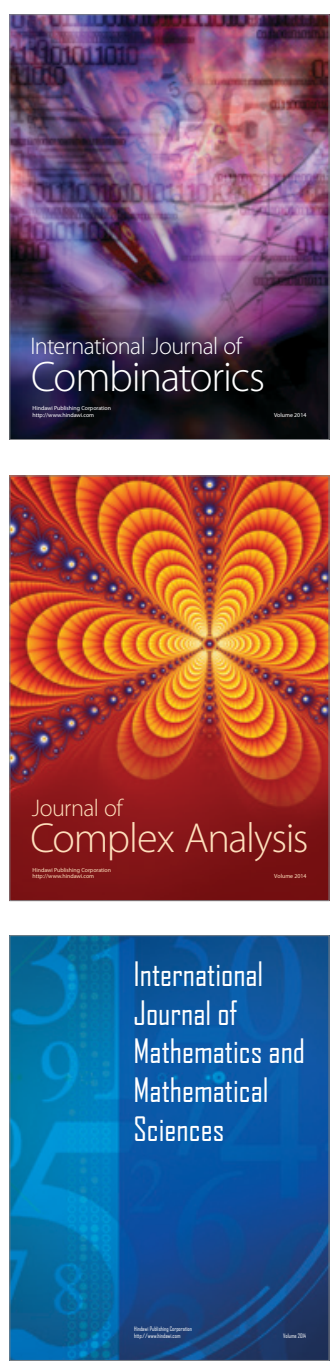
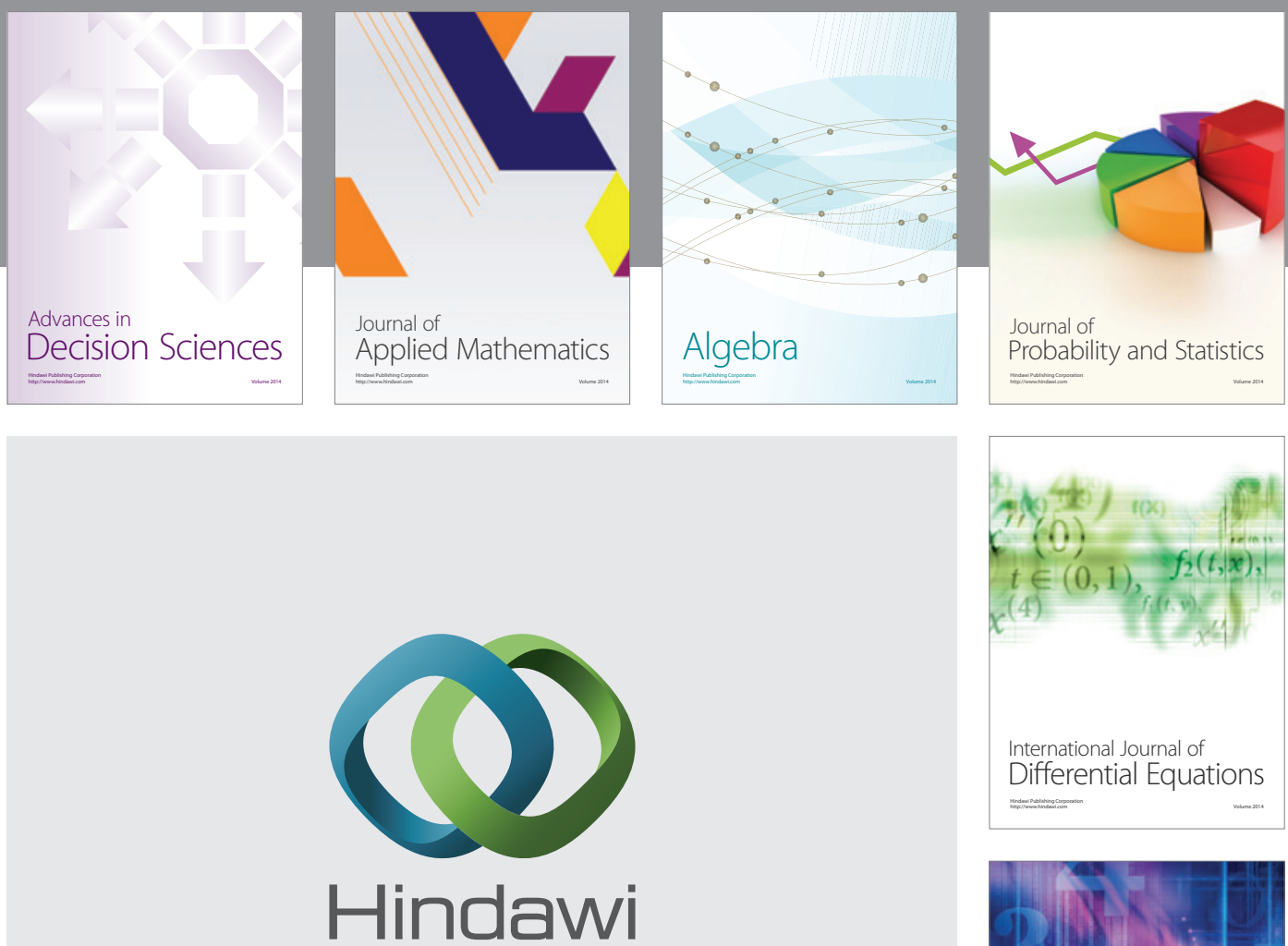

Submit your manuscripts at http://www.hindawi.com
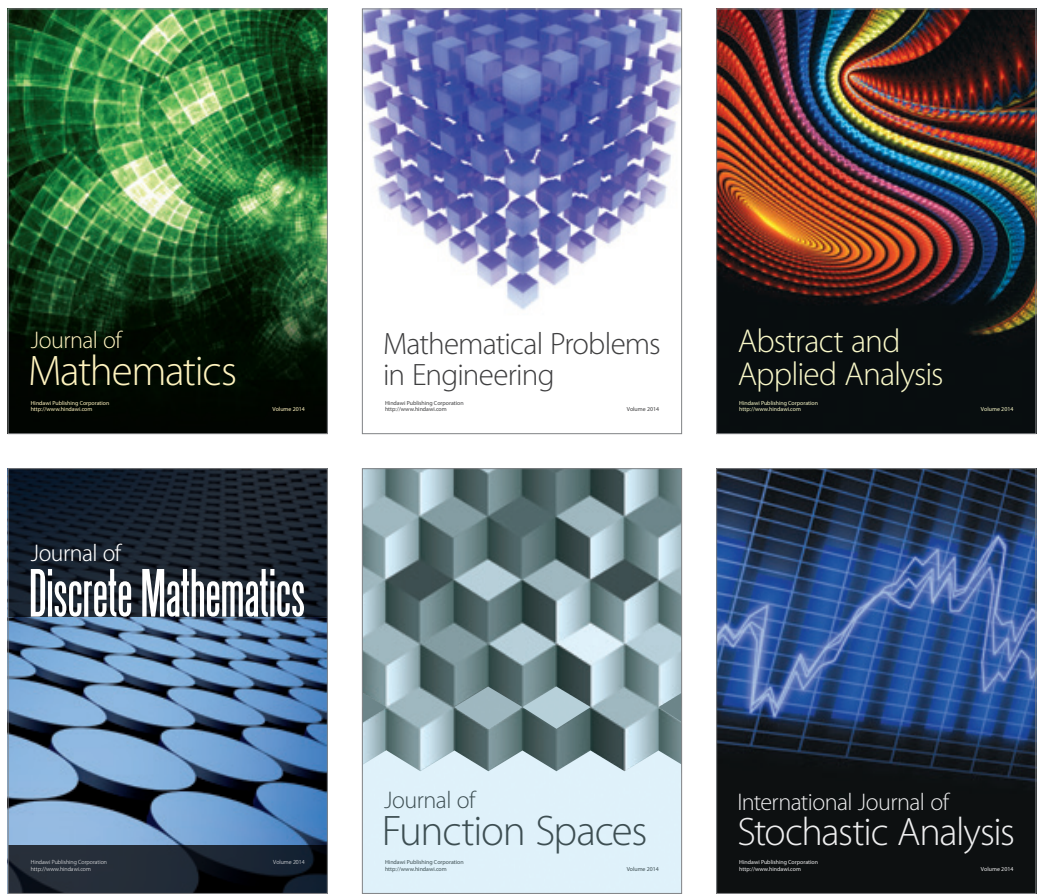

Journal of

Function Spaces

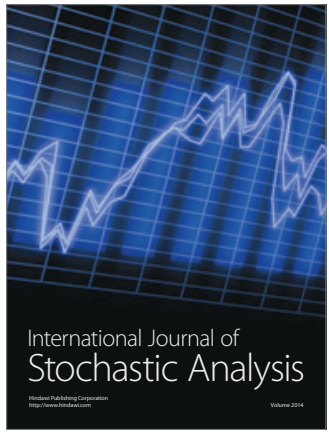

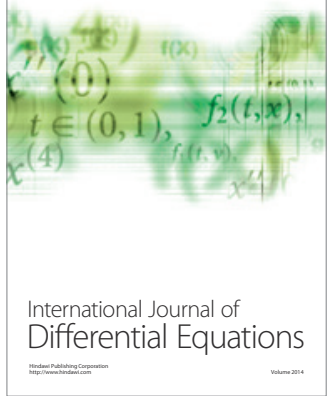
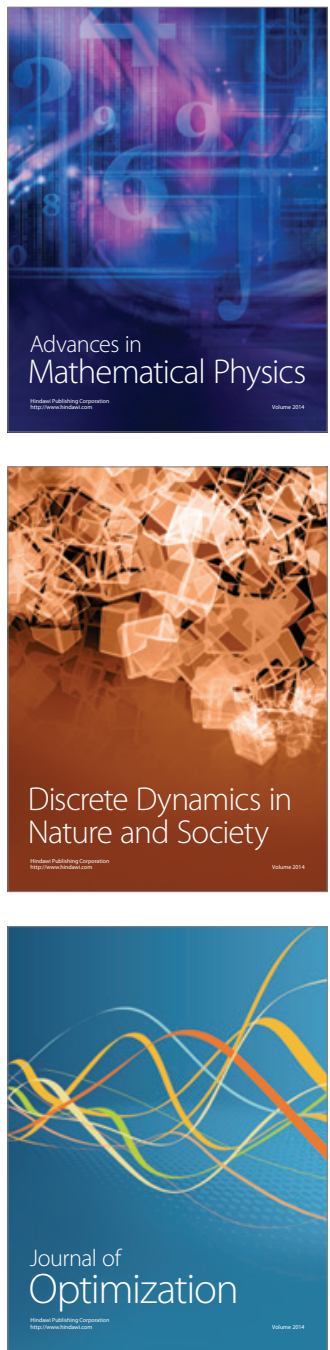